\title{
Minimizing Interference in TDMA MAC Protocols for WSN Operating in Shadow-fading Channels
}

\author{
Mário Macedo, Mário Nunes, and António Grilo
}

\begin{abstract}
This paper presents an analysis of the impact of shadow fading on the performance of TDMA slot allocation, with latter being measured in terms of the resultant in-network interference versus spatial reutilization. Simulations are presented which demonstrate that for Wireless Sensor Networks operating in shadow fading channels, protocols that feature TDMA slot allocation based on channel probing, e.g. LEMMA, lead to a significantly lower interference when compared with protocols based on the $n$-hop neighborhood criterion.
\end{abstract}

Index Terms - Wireless Sensor Networks, TDMA MAC protocols, Slot Allocation, Interference Avoidance, Shadow Fading Channel

\section{INTRODUCTION}

W IRELESS Sensor Networks (WSN) has been a field of increasing research effort in the last years, and several dozens of MAC protocols have been designed for operation on WSNs. The major part of these protocols can be classified as contention-based, or TDMA-based, or even as combinations of these main approaches. Contentionbased protocols present some specific sources of inefficiency such as idle listening, collisions, message overhearing, and control packets overhead. TDMA protocols, on the other hand, are well suited to avoid these problems, but require tight synchronization, and often require complex and sometimes message intensive slot assignment algorithms to guarantee collision and interference free slot schedules.

In this paper, we focus on the slot assignment procedures that are used to avoid collisions and interferences in the communication slots. A large number of TDMA protocols, such as [1], [2], [3], and [4], among others, use an n-hop (usually 2-hops) neighborhood criterion that can be described as following: a node can allocate a slot that is not

Manuscript received November 18, 2007. This work was supported in part by the EuroFGI ("Design and Engineering of the Future Generation Internet"). Apart from this, the European Commission has no responsibility for the content of this paper.

The information in this document is provided as is and no guarantee or warranty is given that the information is fit for any particular purpose. The user thereof uses the information at its sole risk and liability.

M. Macedo, is with INESC-ID, Rua Alves Redol, $n^{\circ}$ 9, Lisboa, Portugal (phone: +351-918293671; fax: +351-213145843; e-mail: mmm@ fct.unl.pt), and UNL/FCT, Caparica, Portugal.

M. Nunes is with INESC-ID, Rua Alves Redol, $\mathrm{n}^{\circ}$ 9, Lisboa, Portugal (phone: +351-213100256; fax: +351-213145843; e-mail: mario.nunes@ inesc-id.pt).

A. Grilo, is with INESC-ID, Rua Alves Redol, no 9, Lisboa, Portugal (phone: +351-213100226; fax: +351-213145843; e-mail: antonio.grilo@ inesc-id.pt). previously occupied by its n-hop neighbors, hoping that this procedure is sufficient to avoid collisions and interferences. However, this approach does not work well in some types of networks, namely for those irregular in shape, as we noted in a previous work [5]. Moreover, this procedure has the disadvantage of being message intensive.

Some other TDMA MAC protocols depart from the $n$-hop neighborhood criterion, but rely on different node transmission powers in order to avoid or identify and reduce possible interferences on the slots. For instance, RID (Radio Interference Detection in Wireless Sensor Networks, see [6]) aims to eliminate possible interference in the slots, by transmitting two detections packets: one transmitted at higher power (HD packet), followed by another transmitted at normal power (ND). With the transmitting node's identification given in the HD packet, and by measuring the power level at the reception of the ND packet, the receiver nodes can therefore estimate the possible interfering nodes. However, these procedures are very complex and computationally intensive.

Motivated by these observations, we designed the LEMMA (Latency-Energy Minimization Medium Access, see [5]) protocol, a new TDMA-based MAC protocol that uses cascading time-slot allocation to minimize latency while still achieving a very low duty-cycle. Although cascading TDMA slot allocation is not new, LEMMA bases slot assignment decisions on the interference really experienced by the WSN nodes through the carrier-sense mechanism. Its slot allocation procedure consists in probing the slots for any activity, both in the father node side, and the child node side, and trying to allocate the slot through messaging exchanges between them, such that other competing pairs for the same slot loose contention and quit allocating the slot.

Moreover, the behavior of the $n$-hop protocols is not studied in the presence of radio irregularity patterns, which are common in real world scenarios. In this paper, we study by simulations, the behavior of the $n$-hop protocols, and of the LEMMA protocol, in the presence of radio irregularity.

\section{RADIO IRREGULARITY PROPAGATION MODELS}

Several authors (c.f., [7] and [8]), argue that the unit radius model, that is commonly used, namely for MAC performance analysis, is simplistic and not accurate, and that the radiation pattern is not circular, but quite irregular in shape. These variations are due to phenomena like reflections, diffraction, and scattering.

A commonly used model that accounts for these effects, 
is the shadowing model referred in [7], which can be described by the following expression:

$P L(d)[d B]=P L\left(d_{0}\right)[d B]+10 \times n \times \log _{10}\left(\frac{d}{d_{0}}\right)+X_{\sigma}$

Where $P L(d)$ is the path loss at distance $d, P L\left(d_{0}\right)$ is the path loss at the reference distance $d_{0}, n$ is the path loss exponent (which values 2 for the free space model), and $X_{\sigma}$ is a random variable, given in $\mathrm{dB}$ units, with a normal distribution of zero mean and standard deviation of $\sigma$. This last term adds irregularity to the radiation pattern, because it introduces a variation from the isotropic pattern of the two first terms of the second half of the expression. Higher standard deviations lead to higher irregularities in the radiation patterns. Several authors, such as [9], measured values of up to $4 \mathrm{~dB}$, for the standard deviation of $X_{\sigma}$, in WSNs scenarios.

Another model that accounts for the anisotropic path losses is RIM (Radio Irregularity Model) presented in reference [8]. In the RIM model, the received signal strength can be modeled by expressions (2) through (4):

RecSignalStrength $=$ SendPower - DOIAdjustedPathLoss + Fading

Where the DOIAdjustedPathLoss (DOI stands for Degree of Irregularity) can be computed as following:

DOIAdjustedPathLoss $=$ PathLoss $\times K_{i}$

$K_{i}$ is a random irregularity factor, valuing around 1 , and $i$ is an integer index variable ranging between 0 and 359, for each degree of the plane. The $K_{i}$ factors are calculated by expression (4):

$k_{i}=1$, if $i=0$

$K i=k_{i-1} \pm$ Rand $\times D O I$, if $0<i<360$,

Where $\left|k_{0}-k_{359}\right| \leq$ DOI must hold.

In expression (4), DOI is an experimentally estimated parameter, which gives the degree of irregularity of a given transmitting node, in some scenario; Rand is a continuous random variable that follows a Weibull distribution; the signs \pm are randomly set.

The authors relate experiences that showed the ability of the RIM model to generate radio patterns that are adequate to model the observed data. One property of this model is that it generates continuous patterns, as the authors say that they are the observed ones. In that paper values are also presented for the DOI parameter, for several MicaZ [13] nodes, which vary between 0.015 , and 0.03 . Adequate Weilbull distribution parameters are also given.

\section{Simulation Model}

This section describes how the behavior of the n-hop neighborhood slot allocation criterion, and of the LEMMA interference avoidance procedure, was assessed in the presence of radio irregularity. As many protocols usually use a 2-hop criterion, the simulations that are presented in this paper were also made for this particular case.

A 100-node square grid was setup in the simulator, with the sink node placed at the upper-right corner.

A tree topology was assumed, with the sink being the root node, and each node communicating with a random neighbor, selected among those that were closest, and that offered more progress towards the sink.

In order to set the distance between the nodes, a result from reference [10] was used, which states that the RSSI (received signal strength), needed to have a high packet receiving rate (PRR), should be higher than $-93 \mathrm{dBm}$, for the MicaZ motes. The minimum received power was thus set to $-90 \mathrm{dBm}$, to allow a safety margin of $3 \mathrm{~dB}$, and the transmitting power of the MicaZ motes was set to the maximum value, $0 \mathrm{dBm}$.

With relation (1), but not considering the random term $X_{\sigma}$, the following expression can be derived:

$d=d_{0} \times 10^{\wedge}\left(\frac{P L(d)-P L\left(d_{0}\right)}{10 \times n}\right)$

The reference distance $d_{0}$ was 1 meter, for which we had a value of $-60 \mathrm{dBm}$ of received power, or a path loss of 60 $\mathrm{dB}$, value that was provided by some previous experiences in our labs, and which agrees with the values that are referred by other authors. Then, with a path loss of $90 \mathrm{~dB}$ for $P L(d)$, expression (5) can be used to estimate the maximum communication range, $d$, which is here designated $d_{\text {comm }}$. For instance, for a path loss exponent of 2 , expression (5) gives a value of $31.6 \mathrm{~m}$ for $d_{\text {comm. The }}$ dimension of the sides of the grid squares was set to $0.85 \times d_{\text {comm }} / \sqrt{2}$, in order to let nodes located diagonally to communicate directly.

In order to estimate the possible interferences, we used a result mentioned in reference [11], which states that for

(4) having a high PRR, near 1, the signal-to-noise ratio (SNR) should be at least $9 \mathrm{~dB}$. Therefore, a value of $-99 \mathrm{dBm}$ (i.e., $-90 \mathrm{dBm}$ for communication, plus $-9 \mathrm{dBm}$ for the signal-tointerference margin) could be set for the threshold of the signal strength, above which it can interfere with the normal communication that is established in the slot.

Expression (5) can also give the minimum distance $\left(d_{\text {not_int }}\right)$ at which another node should be, in order to not interfere to a communication that is established in a given slot, between two nodes that are at a distance of $d_{\mathrm{tx}}$ :

$d_{\text {not_int }} \geq d_{\mathrm{tx}} \times 10^{\wedge}\left(\frac{9 \mathrm{~dB}}{10 \times n}\right)$

For instance, for a path loss exponent $n$ of 2, relation (6) yields $d_{\text {not_int }} \geq 2.8 \times d_{t x}$. This result shows that a 2-hop criterion is enough to avoid interferences in a simple model that does not account for irregularity. For higher path loss exponents, the obtained relations are more favorable, because the signal strengths decay faster. However, this is not true for lower path loss exponents, as we will show later.

Our simulations used the more conventional shadowing log-normal of expression (1). As radio irregularity was included in the model, the criterion that we used for the computation of the 2-hop neighborhood was not geometricbased, but rather communication-based, and it was defined 
as following: the 1-hop neighbors of a given node, are those nodes that can communicate directly with that node; the 2hop neighbors of a given node, are the 1-hop neighbors, plus the nodes that can communicate directly with the 1-hop neighbors.

The simulations began to firstly allocating randomly the slots in a distributed breadth-first manner. The interference avoidance procedure of the 2-hop neighborhood slot allocation criterion consisted in not using the slots that were previously occupied by the 2-hop neighbors of the node, and by the 2-hop neighbors of the father node. For the LEMMA protocol the shadowing model was used along with the interference avoidance procedure. If a pair of nodes, of a father and a child, saw no signal level above the interference level of $-99 \mathrm{dBm}$, when they tested the slot for occupancy, they considered the slot as not occupied. The shadowing model was used to estimate the signal strength at each side of the pair of the father and the child.

Finally, we ran a test to determine if, for all pairs of father and child, there were transmitted signals, coming from another nodes that used the same slot, with strengths such that the $9 \mathrm{~dB}$ signal-to-interference ratio was not respected, both in the father and the child side. In this procedure, the shadowing model was used again in order to estimate these possible interfering signal strengths.

In the simulations, a frame of 1 second of duration, and 68 slots were used. All simulations results were taken from a set of 50 simulation runs.

\section{SimUlation RESULTS AND DISCUSSION}

Fig. 1 shows the average number of interferences that were obtained by the simulation software, for different path loss exponents, and different standard deviations of the lognormal model, and for the 2-hop criterion. And Fig. 2 shows the respective results for the LEMMA interference avoidance slot allocation procedure.

As it can be seen, when the irregularity of the transmission path losses is considered, both slot allocation methods fail to have all the slots free from interference, but the numbers of interferences obtained with the 2-hop criterion are several times higher than those obtained with the LEMMA method. Similar results were also obtained with the RIM model. The LEMMA method presented also superior performance when compared with the 2-hop algorithm.

The number of interferences augments with lower path loss exponents, and with higher standard deviations. If we decrease the path loss exponents, the signals attenuations are lower with the distance, and therefore the irregularity term of the shadowing model can be felt at higher distances.

Referring again to Fig. 1, it can be seen that the 2-hop criterion fails to avoid interferences, even when no irregularity is added (zero for standard deviation), when we have lower path loss exponents (in the case, valuing 1.7). This can be explained by solving expression (6), which yields $d_{\text {not_int }} \geq 3.38 \times d_{\text {tx }}$ for a path loss exponent of 1.7 .

This relation shows that the 2-hop criterion is not enough to avoid interferences, because nodes situated at a distance of $\sqrt{3^{2}+1^{2}} \times d_{\mathrm{tx}}=3.16 \times d_{\mathrm{tx}}$ can still interfere. However, if we lower the signal-to-interference ratio to $8 \mathrm{~dB}$, for which we can expect a PRR around $95 \%$, according to reference [11], the 2-hop criterion would also present no interferences, for the no radio irregularity scenario, because expression (6) would yield $d_{\text {not_int }} \geq 2.96 \times d_{\mathrm{tx}}$.

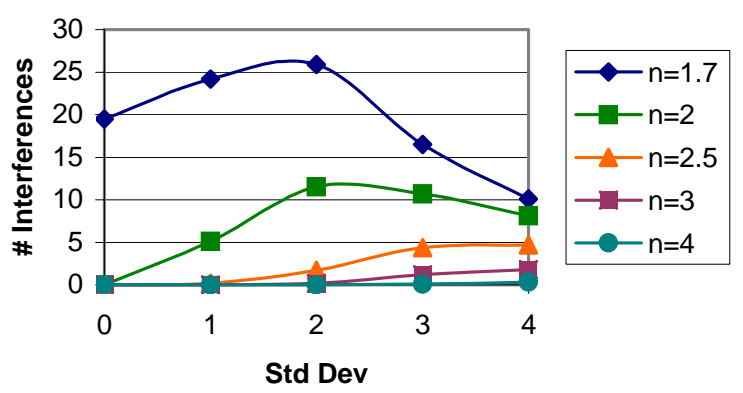

Figure 1. Number of interferences of 2-hop neighborhood slot allocation criterion, when the shadowing model is added.

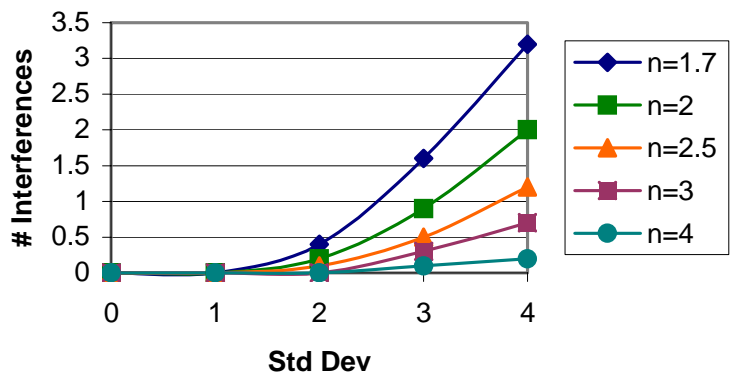

Figure 2. Number of interferences of the LEMMA slot allocation procedure, when the shadowing model is added.

The higher number of interferences that is obtained for the 2-hop criterion seems to be due to a geometric reason: avoiding slots that are used in a 2-hop communication neighborhood is not sufficient in some cases to avoid interferences, since the distance of interference is several times higher than the distance of communication, and not merely twice that value. Adding irregularity seems to worsen even more this effect. When irregularity is added, some transmitted signals can become stronger, or weaker. It is possible that the signal transmitted by one node is not strong enough to make it a 2-hop neighbor of another node, but still being strong enough to interfere with its reception.

Another observed phenomenon is that for lower path loss exponents, the number of interferences experienced by the 2-hop criterion begins to decrease for high values of $\sigma$. The higher number of 2-hop neighbors that are present when the irregularity increases explains this. This last phenomenon is also referred and predicted by mathematical analysis in reference [12], and Fig. 3, and 4 of our simulations corroborate it. But when the neighborhoods of a given node grow, the nodes that could interfere begin to be very far, and therefore have lower probability to do so.

It is also worth to note that higher radio irregularities are usually present for higher path loss exponents (see [12], for this observation). Therefore, the highest values of interferences that were obtained for the basic LEMMA protocol simulations, and shown in the upper-right side of 
Fig. 2 - which were present in the scenarios with higher values of $\sigma$ and with the lower path loss exponent values of 1.7 , and 2 - correspond to situations that are probably more rare. This argument supports also the conclusion that the basic LEMMA protocol is much better than the 2-hop criterion in terms of number of resulting interferences.

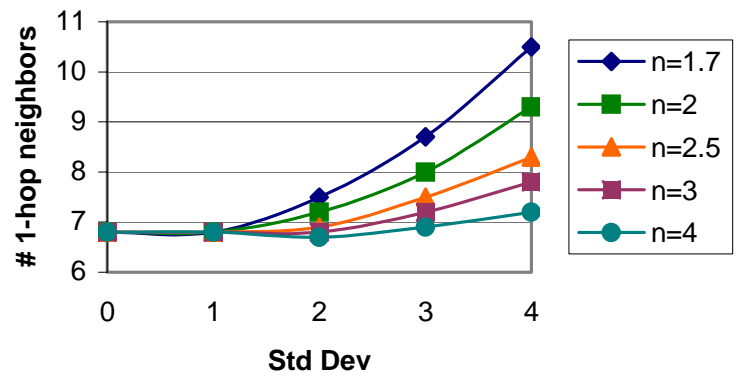

Figure 3. Number of 1-hop neighbors obtained for different path loss exponents and irregularities.

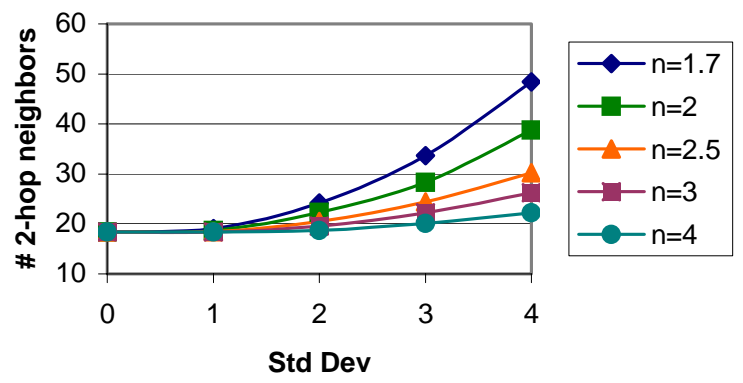

Figure 4. Number of 2-hop neighbors obtained for different path loss exponents and irregularities.

Regarding to the interferences that are present with the LEMMA protocol, they result from the asymmetry of the links: suppose that a pair of father and child had previously allocated a slot, and another pair of father and child achieve to allocate the same slot, because they do not feel it as occupied. Due to asymmetry of the transmissions, that arises when radio irregularity is added, this last pair can interfere with the communications of the former pair.

We are working on a solution to this problem. It consists in adding tones, to the basic LEMMA protocol allocation messages. These tones are transmitted at a higher power level than that of the protocol messages, and were proved to be able to overcome the asymmetry of the links, leading to almost zero interferences.

\section{CONCLUSIONS AND FUTURE WORK}

This paper has presented a comparison between TDMA slot allocation based on LEMMA, and protocols that employ the $n$-hop neighborhood criterion. Simulations lead to the conclusion that in real environments, the LEMMA protocol will show a substantial advantage over the more commonly used 2-hop neighborhood criterion, in terms of number of interferences that persist after slot allocation. Interferences in the LEMMA protocol are due to asymmetric links, while interferences in the 2-hop criterion are also inherent to its geometric nature. The 2-hop neighborhood slot allocation criterion presents an intolerable number of interferences when a more realistic radio propagation model is considered, and when a realistic signal-to-interference ratio is used. This criterion also presents problems when more irregular shape networks are considered.

We also presented an extension to the basic LEMMA that achieves almost zero interferences.

For future work, the authors intend to continue this study with the application of more sophisticated radio propagation models, such as by adding spatial correlation to the shadowfading model.

\section{REFERENCES}

[1] L. Bao, J. J. Garcia-Luna-Aceves, "A New Approach to Channel Access Scheduling for Ad Hoc Networks," Proceedings of the Seventh Annual International Conference on Mobile Computing and Networking, ACM MobiCom 2001, Rome, Italy, July 2001.

[2] V. Rajendran, K. Obraczka, J. J. Garcia-Luna-Aceves, "EnergyEfficient, Collision-Free Medium Access Control for Wireless Sensor Networks", Proceedings of the ACM SenSys 2003. Los Angeles, USA, 2003.

[3] V Rajendran, J. J. Garcia-Luna-Aceves, K. Obraczka, "EnergyEfficient, Application-Aware Medium Access for Sensor Networks", Proceedings of 2nd IEEE International Conference on Mobile Ad-Hoc and Sensor Systems, IEEE MASS 2005, Washington, USA, November 2005.

[4] L. van Hoesel, P. Havinga, "A Lightweight Medium Access Protocol (LMAC) for Wireless Sensor Networks", Proceedings of the First International Workshop on Networked Sensing Systems (INSS04), Tokyo, Japan, June 2004.

[5] M. Nunes, A. Grilo, M. Macedo, "Interference-Free TDMA Slot Allocation in Wireless Sensor Networks", Proceedings of the 32nd IEEE Conference on Local Computer Networks (LCN 2007), pp. 239241, Dublin, Ireland, October 2007

[6] G. Zhou, T. He, J. A. Stankovic, T. Abdlezaher, "RID: Radio Interference Detection in Wireless Sensor Networks", Proceedings of the 24th Annual Joint Conference of the IEEE Computer and Communications Societies (INFOCOM 20005), Miami, USA; March 2005.

[7] T. Rappaport, "Wireless Communications: Principles and Practice", 2nd Edition, Prentice Hall, 2002.

[8] G. Zhou, T. He, S. Krishnamurthy, J. A. Stankovic, "Models and Solutions for Radio Irregularity in Wireless Sensor Networks", ACM Transactions on Sensor Networks, Volume 2, Issue 2, pp. 221-262, May 2006.

[9] A. Martinez-Sala, J.M. Molina-Garcia-Pardo, E. Egea-Lopez, J. Vales-Alonso, L. Juan-Llacer, J. Garcia-Haro, "An Accurate Radio Channel Model for Wireless Sensor Networks Simulation”, Journal of Communications and Networks, Vol. 7, Part 4, pp. 401-407, The AIEI KICS, South Korea, 2005.

[10] K. Srinivasan, P. Levis, "RSSI is Under Appreciated", Proceedings of the Third Workshop on Embedded Networked Sensors (EmNets 2006), Harvard University, Cambridge, MA, USA, May 2006.

[11] H. Lee, A. Cerpa, P. Levis, "Improving Wireless Simulation through Noise Modeling", Proceedings of the 6th International Conference on Information Processing in Sensor Networks (IPSN '07), Cambridge, Massachusetts, USA, April 2007.

[12] C. Bettstetter, C. Hartmann, "Connectivity of Wireless Multihop Networks in a Shadow Fading Environment", Wireless Networks, Volume 11, Number 5, pp. 571-579, Springer Netherlands, September 2005.

[13] "MICAz datasheet", Crossbow, http://www.xbow.com/Products/ productdetails.aspx?sid=164, November $16^{\text {th }} 2007$ (date of last access). 